\title{
The effect of low dose glargine U 300 on uncontrolled type 2 diabetes mellitus. An observational study in Indian patients
}

\section{ABSTRACT}

Introduction. Insulin therapy plays an important role in the management of diabetes mellitus. The primary goal of insulin therapy is to achieve the best possible glycemic control without hypoglycemia. First-generation basal insulin (BI) analogues, such as insulin glargine $100 \mathrm{U} / \mathrm{ml}$ (Gla-100) and insulin detemir (IDet), provide more prolonged and stable activity than neutral protamine Hagedorn (NPH) insulin, with a lower risk of hypoglycemia. Insulin glargine $300 \mathrm{U} / \mathrm{mL}$ (glargine $\mathrm{U}$ 300) is a long acting basal insulin analogue approved for the treatment of diabetes mellitus. Insulin glargine $300 \mathrm{U} / \mathrm{mL}$ has a more stable and prolonged pharmacokinetic/ /pharmacodynamics profile than insulin glargine $100 \mathrm{U} / \mathrm{mL}$, with a duration of glucose-lowering activity exceeding $\mathbf{2 4} \mathrm{h}$. Although the average daily insulin dose was higher, hypoglycemia episodes were lower in patients treated with Gla-300 compared with those treated with Gla-100. This is due to a more extended time action profile than Gla-100 resulting in a more stable and sustained glycemic control. The formulation of Gla-100 delivers the same amount of Insulin as Gla-300, in a third of the injection volume. It is essential to determine whether the clinical benefits of hypoglycemia reduction observed with insulin glargine in RCTs translate into a real-life clinical practice setting.

Address for correspondence:

Dr. Asis Mitra

Ruby General Hospital

Kasba Golpark, 700107 Kolkata, India

e-mail: asis.mitra10@gmail.com

Clinical Diabetology 2020, 9, 2, 128-133

DOI: $10.5603 /$ DK.2020.0010

Received: 26.12 .2019

Accepted: 19.01.2020
Materials and methods. Fifty patients diagnosed with type 2 diabetes mellitus with uncontrolled plasma glucose levels $\left(\mathrm{HbA}_{1 \mathrm{c}}\right.$ of $>$ 7.5) who were on oral hypoglycemic agents, premix insulin and basal bolus therapy were enrolled into the study and started on glargine $U \mathbf{3 0 0}$ at a dose of $0.2 \mathrm{lU} / \mathrm{kg}$ and analysed for glycemic and kidney function parameters. The patients were followed up at 3 and 6 months post treatment. Results. All glycemic control parameters decreased significantly with almost a $50 \%$ decline in both FBS and PBS from baseline to 6 months. The $\mathrm{HbA}_{1 \mathrm{c}}$ decreased significantly from baseline to 3 months and 6 months post treatment by $18 \%$ and $29 \%$ respectively. All biochemical parameters were found to be statistically significant in both groups.

Conclusions. In patients with uncontrolled type 2 diabetes, switching from either OHAs or insulin to Gla-300 improves glycemic control, with a low incidence of hypoglycemia. These results confirm the effectiveness and safety of Gla-300 in a real-world setting and show that Gla-300 is a suitable therapy option for patients with diabetes. (Clin Diabetol 2020; 9; 2: 128-133)

Key words: type 2 diabetes mellitus, insulin analogues, glargine $U$ 300, hypoglycemia, kidney function parameters, $\mathrm{HbA}_{1 \mathrm{c}^{\prime}}$ fasting blood sugar, post prandial blood sugar

\section{Introduction}

Diabetes mellitus is a metabolic disease marked by elevated plasma glucose. In type 2 diabetes mellitus (T2DM), the pancreas often continues to produce insulin, sometimes even at higher-than-normal levels, 
especially early in the course of the disease. However, the body develops resistance to the effects of insulin, so there is not enough insulin to meet the body's needs. As type 2 diabetes progresses, the insulin-producing ability of the pancreas decreases. T2DM tends to develop in adults, and it results from insulin resistance accompanied by a gradual decline in $\beta$-cell function. In T1 and T2DM, impaired glucose regulation leads to chronic hyperglycemia, which induces a variety of vascular complications.

The global diabetes epidemic has reached alarming proportions, particularly in Asia. In 2017, prevalence among adults ranged from $7.7 \%$ to $13.7 \%$ in this region with more than 1 million diabetes-related deaths [1].

Although there is no cure for diabetes yet, it can be treated and effectively controlled. The goals of diabetes management are to keep the plasma glucose levels as near to optimal levels as possible by balancing diet with medication and activity. Other co-morbidities such as hypercholesteremia, hypertension and dyslipidemia should also be controlled. Despite the introduction of numerous anti-hyperglycemic medications, many patients with T2DM require insulin, and basal insulins continue to be frequently used either as first-line insulin treatment or as part of multiple daily injection regimens. Current American Diabetes Association (ADA) guidelines recommend a $\mathrm{HbA}_{1 \mathrm{c}}$ target of $7.0 \%$ [2].

Insulin therapy plays an important role in the management of diabetes mellitus. The primary goal of insulin therapy is to achieve the best possible glycemic control without hypoglycemia. First-generation basal insulin (BI) analogues, such as insulin glargine $100 \mathrm{U} / \mathrm{ml}$ (Gla-100) and insulin detemir (IDet), provide more prolonged and stable activity than neutral protamine Hagedorn (NPH) insulin, with a lower risk of hypoglycemia [3]. It is essential to determine whether the clinical benefits of hypoglycemia reduction observed with insulin glargine in RCTs translate into a real-life clinical practice setting.

Insulin glargine $300 \mathrm{U} / \mathrm{mL}$ (glargine $\mathrm{U} 300$ ) is a long acting basal insulin analogue approved for the treatment of diabetes mellitus. Insulin glargine $300 \mathrm{U} / \mathrm{mL}$ has a more stable and prolonged pharmacokinetic/ /pharmacodynamics profile than insulin glargine $100 \mathrm{U} / \mathrm{mL}$, with a duration of glucose-lowering activity exceeding $24 \mathrm{~h}$. Clinical trial data has shown that, following injection, Gla-300 has slower rate of release into the surrounding tissues when compared with Gla-100. This results in a more even plasma concentration of the drug and also better glucose lowering effect resulting in a longer duration of action that fully covers a 24-h dose period with a single injection [4].

The efficacy and safety of Gla-300 was studied extensively in the EDITION series of clinical trials, which compared Gla-300 with Gla-100 in patients with T1D or T2D, with differing treatment backgrounds. These clinical trials demonstrated that hemoglobin $A_{1 c}$ level decreased by equivalent amounts with Gla-300 and Gla-100 treatment, regardless of the type of diabetes or whether patients were insulin naive or being switched from oral treatment with another basal insulin.

The most common and serious adverse effect of insulin treatment, hypoglycemia remains a key unmet medical need in insulin-treated diabetes. Insulin related hypoglycemia and insulin-use errors account for a significant number of hospital visits. The risk of hypoglycemic episodes increase in the elderly and patients with renal impairment. Although the average daily insulin dose was higher, hypoglycemia episodes were lower in patients treated with Gla-300 compared with those treated with Gla-100 $[5,6]$. This is due to a more extended time action profile than Gla-100 resulting in a more stable and sustained glycemic control. The formulation of Gla-100 delivers the same amount of Insulin as Gla-300, in a third of the injection volume.

The current study was envisaged to evaluate the effects of Gla-300 when prescribed in uncontrolled diabetes including to assess effects on markers of kidney function along with parameters determining glycemic control over a period of six months prompting the need for a more careful management of insulin therapy.

\section{Materials and methods}

This was an observational study. 50 patients diagnosed with type 2 diabetes mellitus with uncontrolled plasma glucose levels $\left(\mathrm{HbA}_{1 \mathrm{c}}\right.$ of $\left.>7.5\right)$ who were on oral hypoglycemic agents premix insulin or basal bolus regime, were enrolled into the study. Initially all subjects were started on glargine $U 300$ at a dose of $0.2 \mathrm{IU} / \mathrm{kg}$. Patients treated in diabetes clinic of Ruby General Hospital and principal investigator had complete oversight of the patients during the six month study period. The dose was titrated up to $0.4 \mathrm{lU} / \mathrm{kg}$ over a period of two weeks according to self-monitoring for blood glucose (SMBG) diary. Patients who were on premix insulin were converted to basal bolus regime with the basal insulin being glargine $\mathrm{U} 300$ and bolus insulin with human insulin Actrapid. In patients who were already on basal bolus insulin their basal insulin was changed to glargine U300, keeping bolus dose unaltered. Patients were instructed to do SMBG per day and maintain a diary. The 5 SMBGs recommended were 3 premeals, 1 bedtime and 1 at 3 am and SOS measurement if the subjects experienced an episode of hypoglycaemia. Hypoglycemia was defined as glucose levels below $70 \mathrm{mg} / \mathrm{dL}$. Patients were instructed to look out for symptoms suggestive of hypoglycemia 
Table 1. Demographics of the study population

\begin{tabular}{lccc}
\hline & All subjects $(\mathbf{N}=\mathbf{4 8})$ & Male $(\mathbf{N}=\mathbf{2 5})$ & Female $(\mathbf{N}=\mathbf{2 3})$ \\
\hline Age in years (mean) & $58.42 \pm 9.65$ & $60.4 \pm 10.79$ & $56.26 \pm 7.92$ \\
Height $[\mathrm{cm}]$ & $162.94 \pm 9.22$ & $166.84 \pm 8.35$ & $158.7 \pm 8.34$ \\
Weight $[\mathrm{kg}]$ & $66.35 \pm 11.67$ & $69.76 \pm 11.65$ & $62.65 \pm 10.74$ \\
BMI $\left[\mathrm{kg} / \mathrm{m}^{2}\right]$ & $25.03 \pm 3.99$ & $25.19 \pm 4.3$ & $24.84 \pm 3.73$
\end{tabular}

$\mathrm{BMI}$ - body mass index

Table 2. Comparison of changes in biochemical parameters in 48 subjects at baseline, 3 months and 6 months follow up

\begin{tabular}{|c|c|c|c|c|c|c|c|c|}
\hline $\begin{array}{l}\text { Age } \\
(N=48)\end{array}$ & BMI & Parameters & Baseline & 3 months & 6 months & $\begin{array}{c}\text { Change in } \\
3 \text { months (\%) }\end{array}$ & $\begin{array}{c}\text { Change in } \\
6 \text { months (\%) }\end{array}$ & P-value \\
\hline \multirow[t]{5}{*}{$58.42 \pm 9.65$} & $25.02 \pm 4$ & ACR & $46.5 \pm 44.1$ & $33.54 \pm 27.82$ & $24.31 \pm 17.79$ & -27.87 & -47.72 & 0.00003 \\
\hline & & eGFR & $78.06 \pm 13.54$ & $81.33 \pm 12.44$ & $83.58 \pm 12.36$ & 4.19 & 7.07 & $<0.00001$ \\
\hline & & FBS & $213.73 \pm 71.92$ & $138.75 \pm 29.66$ & $112.04 \pm 25.25$ & -35.08 & -47.58 & $<0.00001$ \\
\hline & & PPBS & $345.46 \pm 91.63$ & $229.38 \pm 49.25$ & $178.73 \pm 21.69$ & -33.60 & -48.26 & $<0.00001$ \\
\hline & & $\mathrm{HbA}_{1 \mathrm{c}}$ & $10.03 \pm 0.91$ & $8.24 \pm 0.67$ & $7.1 \pm 0.24$ & -17.88 & -29.28 & $<0.00001$ \\
\hline
\end{tabular}

$\mathrm{BMI}$ - body mass index; ACR — albumin creatinine ratio in spot urine sample; eGFR - estimated glomerular filtration rate by CKD-EPI formula; FBS - fasting blood sugar; PPBS -2 hour post prandial blood sugar

such as dizziness, excessive sweating, reeling of head, confusion. The target glucose levels were between 140-180 mg/dL. Glargine U 300 was titrated to a target pre-prandial glucose concentration of $100-130 \mathrm{mg} / \mathrm{dL}$ and postprandial glucose concentration of 140$-180 \mathrm{mg} / \mathrm{dL}$ based on CBG levels assessed on a weekly basis for two weeks at the clinic. Patients with type 1 diabetes, pregnant or lactating mothers, allergic to insulin or having estimated glomerular filtration rate (eGFR) of $\leq 30$ were excluded. Demographic details, details of diabetes such as duration, treatment and diabetes education received etc. were collected from the patients. Two subjects were lost to follow up during the course of the study. One subject died due to bronchogenic carcinoma which was not related to insulin. The other subject dropped out because she could not afford the expenses involved in the therapy. The primary objective of the study was to evaluate the change in the parameters of fasting blood sugar, post prandial blood sugar and $\mathrm{HBA}_{1 \mathrm{c}}$ from baseline to 3 months and 6 months post treatment. The secondary objective was to evaluate the change in the parameters of biochemical markers of renal function from baseline to 3 months and 6 months post treatment. The safety objective was to evaluate the episodes of hypoglycaemia during the study period.

This study was carried out on FDA approved insulin analogue among Indian patients with diabetes. This study was conducted in accordance to the Declaration of Helsinki (1975 as revised in 2013).

\section{Statistical analysis}

All data analysis was carried out as per a comprehensive pre-planned statistical analysis plan. The intention-to treat analysis of the primary study end point was performed on all randomly allocated participants who satisfactorily complete all follow up visits. To evaluate the overall effects of glargine $U 300$ on markers of glycemic control and chronic kidney disease, the mean changes from baseline to 3 months and 6 months post treatment is calculated. P-value 0.05 was defined as the level of statistical significance. Data is expressed as mean \pm SD for continuous variables and as percentage for categorical variables.

\section{Results}

Table 1 summarizes the demographic characteristics of the patients. The average age of the subjects in the study was $58.42 \pm 9.65$ years. The average age of males were higher compared to the females $(60.4 \pm 10.79 \mathrm{vs}$. $56.26 \pm 7.92$ years). The average height of subjects in the study was $162.94 \pm 9.22 \mathrm{~cm}$ with males comparatively taller than females $(166.84 \pm 8.35$ vs. $158.7 \pm$ $\pm 8.34 \mathrm{~cm}$ ). The average weight of subjects in the study was $66.35 \pm 11.67 \mathrm{~kg}$ with males comparatively heavier than females $(69.76 \pm 11.65$ vs. $62.65 \pm 10.74 \mathrm{~kg})$. The average BMI of the subjects was $25.03 \pm 3.99$.

Table 2 summarizes the biochemical parameters (ACR, eGFR, FBS, PBS and $\mathrm{HbA}_{1 \mathrm{c}}$ ) for all enrolled patients compared from baseline to the third month and the sixth month using repeated measures ANOVA and 
Table 3. Baseline characteristics by categories of ACR ( $<30 \mathrm{mg} / \mathrm{g}$ i.e., normal) irrespective of BMI status and \% change after treatment received

\begin{tabular}{|c|c|c|c|c|c|c|c|c|}
\hline Age & BMI & $\begin{array}{c}\text { Parameters } \\
\text { N }=23\end{array}$ & Baseline & 3 months & 6 months & $\begin{array}{c}\text { Change in } \\
3 \text { months (\%) }\end{array}$ & $\begin{array}{l}\text { Change in } \\
6 \text { months (\%) }\end{array}$ & P-value \\
\hline \multirow[t]{5}{*}{$59.48 \pm 10.14$} & $24.35 \pm 3.24$ & $A C R$ & $17.48 \pm 5.98$ & $25.57 \pm 27.51$ & $13.13 \pm 4.59$ & 46.28 & -24.89 & 0.0355 \\
\hline & & eGFR & $75.26 \pm 11.1$ & $79.74 \pm 9.88$ & $82.35 \pm 10.46$ & 5.95 & 9.42 & $<0.00001$ \\
\hline & & FBS & $204.43 \pm 49.71$ & $138.65 \pm 34.10$ & $113.96 \pm 33.66$ & -32.18 & -44.25 & $<0.00001$ \\
\hline & & PPBS & $328.91 \pm 85.93$ & $227.09 \pm 49.2$ & $177.26 \pm 27.32$ & -30.96 & -46.11 & $<0.00001$ \\
\hline & & $\mathrm{HbA}_{1 \mathrm{c}}$ & $9.88 \pm 0.64$ & $8.02 \pm 0.52$ & $7.05 \pm 0.18$ & -18.83 & -28.64 & $<0.00001$ \\
\hline
\end{tabular}

BMI - body mass index; ACR - albumin creatinine ratio in spot urine sample; eGFR - estimated glomerular filtration rate by CKD-EPI formula; FBS - fasting blood sugar; PPBS -2 hour post prandial blood sugar

Table 4. Baseline characteristics by categories of ACR (30-300 mg/g i.e., microalbuminuria) irrespective of BMI status and $\%$ change after treatment received

\begin{tabular}{|c|c|c|c|c|c|c|c|c|}
\hline Age & BMI & $\begin{array}{c}\text { Parameters } \\
\mathrm{N}=25\end{array}$ & Baseline & 3 months & 6 months & $\begin{array}{c}\text { Change in } \\
3 \text { months (\%) }\end{array}$ & $\begin{array}{c}\text { Change in } \\
6 \text { months (\%) }\end{array}$ & P-value \\
\hline \multirow[t]{5}{*}{$57.44 \pm 9.28$} & $25.64 \pm 4.57$ & ACR & $73.2 \pm 47.19$ & $40.88 \pm 26.55$ & $34.6 \pm 19.25$ & -44.15 & -52.73 & $<0.00001$ \\
\hline & & eGFR & $80.64 \pm 15.23$ & $82.8 \pm 14.46$ & $84.72 \pm 14$ & 2.68 & 5.06 & 0.0006 \\
\hline & & FBS & $222.28 \pm 87.77$ & $138.84 \pm 25.63$ & $110.28 \pm 14.26$ & -37.54 & -50.39 & $<0.00001$ \\
\hline & & PPBS & $360.68 \pm 95.76$ & $231.48 \pm 50.21$ & $180.08 \pm 15.28$ & -35.82 & -50.07 & $<0.00001$ \\
\hline & & $\mathrm{HbA}_{1 \mathrm{c}}$ & $10.18 \pm 1.1$ & $8.44 \pm 0.73$ & $7.14 \pm 0.28$ & -17.09 & -29.86 & $<0.00001$ \\
\hline
\end{tabular}

$\mathrm{BMI}$ - body mass index; ACR - albumin creatinine ratio in spot urine sample; eGFR - estimated glomerular filtration rate by CKD-EPI formula; FBS - fasting blood sugar; PPBS -2 hour post prandial blood sugar

were found to be statistically significant. There was a reduction of $27 \%$ and $47 \%$ in albumin creatinine ratio from baseline to 3 months and 6 months respectively. Similarly the eGFR increased by $4 \%$ and $7 \%$ from baseline to 3 months and 6 months respectively. All glycemic control parameters decreased significantly with a $47 \%$ and $48 \%$ decline in FBS and PPBS from baseline to 6 months respectively. The $\mathrm{HbA}_{1 \mathrm{c}}$ decreased significantly from baseline to 3 months and 6 months post treatment by $17 \%$ and $29 \%$ respectively.

Subjects were categorized into mildly increased and moderately increased groups based on the ACR values, assuming a cut of level of $(<30 \mathrm{mg} / \mathrm{g})$ irrespective of their BMI status with 23 and 25 subjects respectively. All biochemical parameters were found to be statistically significant in both groups. Although there was an increase of $46 \%$ in albumin creatinine ratio from baseline to 3 months the values decreased at 6 months. Similarly the eGFR increased by $5 \%$ and $9 \%$ from baseline to 3 months and 6 months respectively. All glycemic control parameters decreased significantly with a $44 \%$ and $46 \%$ decline in both FBS and PBS from baseline to 6 months. The $\mathrm{HbA}_{1 \mathrm{c}}$ decreased significantly from baseline to 3 months and 6 months post treatment by $18 \%$ and $28 \%$ respectively (Table 3 ).
Biochemical parameters for ACR levels of moderately increased groups irrespective of BMI status were evaluated. There were statistically significant differences in all parameters from baseline to 3 months and 6 months (Table 4).

Biochemical parameters for overweight subjects according to the BMI were analyzed for ACR levels of moderately increased groups. Although there were statistically significant differences in all parameters from baseline to 3 months and 6 months, the eGFR in the moderately increased group was found to be statistically not significant (Table 5).

Comparison of parameters in subjects receiving Insulin along with oral hypoglycemic agents (OHAs) is presented above. The biochemical parameter of ACR and eGFR along with glycemic parameters $\mathrm{HbA}_{1 \mathrm{c}}$ and FBS and PBS reduced from baseline to study end and this reduction was statistically significant (Table 6).

The number of subjects at various levels of intensity of risk for CKD were analyzed considering both eGFR and UACR values. It was found that there was a decline in the number of subjects in the moderately increased risk by the end of the study, whereas the number of subject in the low risk category increased from 21 to 38 towards the end of 6 months of the study (Table 7). 
Table 5. Baseline characteristics by categories of ACR (30-300 mg/g i.e., microalbuminuria) and BMI status of overweight and \% change after treatment received

\begin{tabular}{|c|c|c|c|c|c|c|c|c|}
\hline Age & BMI & $\begin{array}{c}\text { Parameters } \\
\mathrm{N}=13\end{array}$ & Baseline & 3 months & 6 months & $\begin{array}{c}\text { Change in } \\
3 \text { months (\%) }\end{array}$ & $\begin{array}{l}\text { Change in } \\
6 \text { months (\%) }\end{array}$ & P-value \\
\hline \multirow[t]{5}{*}{$57.54 \pm 9.97$} & $27.92 \pm 1.26$ & $A C R$ & $89.85 \pm 57.36$ & $50.62 \pm 33.15$ & $41.77 \pm 20.7$ & -43.66 & -53.51 & 0.0003 \\
\hline & & eGFR & $84.92 \pm 13.33$ & $86.77 \pm 12.13$ & $88.77 \pm 12.45$ & 2.18 & 4.53 & 0.1128 \\
\hline & & FBS & $192.23 \pm 43.32$ & $130 \pm 19.85$ & $105.85 \pm 9.81$ & -32.37 & -44.94 & $<0.00001$ \\
\hline & & PPBS & $347.69 \pm 52.57$ & $222.08 \pm 22.86$ & $181.08 \pm 11.82$ & -36.13 & -47.92 & $<0.00001$ \\
\hline & & $\mathrm{HbA}_{1 \mathrm{c}}$ & $10.08 \pm 0.46$ & $8.43 \pm 0.33$ & $7.15 \pm 0.2$ & -16.37 & -29.07 & $<0.00001$ \\
\hline
\end{tabular}

$\mathrm{BMI}$ - body mass index; ACR — albumin creatinine ratio in spot urine sample; eGFR — estimated glomerular filtration rate by CKD-EPI formula; FBS — fasting blood sugar; PPBS -2 hour post prandial blood sugar

Table 6. Comparison of biochemical parameters in insulin (once daily glargine U 300) + oral hypoglycemic agents (OHAs) group at baseline, 3 months and 6 months follow up

\begin{tabular}{|c|c|c|c|c|c|c|c|c|}
\hline Age & BMI & $\begin{array}{c}\text { Parameters } \\
\mathrm{N}=43\end{array}$ & Baseline & 3 months & 6 months & $\begin{array}{c}\text { Change in } \\
3 \text { months (\%) }\end{array}$ & $\begin{array}{c}\text { Change in } \\
6 \text { months (\%) }\end{array}$ & P-value \\
\hline \multirow[t]{5}{*}{$58.42 \pm 9.65$} & $25.02 \pm 4$ & $A C R$ & $46.5 \pm 44.1$ & $33.54 \pm 27.82$ & $24.31 \pm 17.79$ & -27.87 & -47.72 & 0.00003 \\
\hline & & eGFR & $78.06 \pm 13.54$ & $81.33 \pm 12.44$ & $83.58 \pm 12.36$ & 4.19 & 7.07 & $<0.00001$ \\
\hline & & FBS & $213.73 \pm 71.92$ & $138.75 \pm 29.66$ & $112.04 \pm 25.25$ & -35.08 & -47.58 & $<0.00001$ \\
\hline & & PPBS & $345.46 \pm 91.63$ & $229.38 \pm 49.25$ & $178.73 \pm 21.69$ & -33.60 & -48.26 & $<0.00001$ \\
\hline & & $\mathrm{HbA}_{1 \mathrm{c}}$ & $10.03 \pm 0.91$ & $8.24 \pm 0.67$ & $7.1 \pm 0.24$ & -17.88 & -29.28 & $<0.00001$ \\
\hline
\end{tabular}

$\mathrm{BMI}$ - body mass index; ACR - albumin creatinine ratio in spot urine sample; eGFR — estimated glomerular filtration rate by CKD-EPI formula; FBS - fasting blood sugar; PPBS -2 hour post prandial blood sugar

Table 7. Comparison of no of subjects in term of risk at baseline, 3 months and 6 months follow up [7]

\begin{tabular}{lccc}
\hline Intensity of risk & Baseline & $\mathbf{3}$ Months & $\mathbf{6}$ months \\
\hline Low risk & $21(43.75 \%)$ & $28(58.33 \%)$ & $38(79.17 \%)$ \\
Moderately increased risk & $27(56.25 \%)$ & $20(41.67 \%)$ & $10(20.83 \%)$ \\
High risk & 0 & 0 & 0 \\
Very high risk & 0 & 0 & 0 \\
\hline
\end{tabular}

There were $5(10 \%)$ patients who reported episodes of hypoglycaemia at various time points in the course of the study. None of the patients required hospitalization and were managed with oral glucose solution. The dose of their OHA or insulin was reduced.

\section{Discussion}

This observational study evaluated the efficacy and safety of glargine $U \mathbf{3 0 0}$ in patients with uncontrolled T2DM on oral hypoglycemic agents and/or insulin therapy. The subjects were switched to Gla-U 300 as they failed to respond to current line of management due to less than optimal glycemic control. The study aims to explore the effectiveness and safety of Gla-U 300 in eastern Indian population. All patients were initially started on a low dose of 0.2 units as against the western standard of 0.4 units, the rationale behind this approach is the lower BMI observed in Indian population. After adequate titration the patients were followed up at 3 months and 6 months. Glycemic control was significantly improved with a very low incidence rate of hypoglycemia and no significant weight change.

The switch over from basal/bolus insulin or oral hypoglycemic agents to Gla-300 has been studied in previous trials. The EDITION-2, a phase 3a randomized controlled trial and DELIVER-2 was a retrospective cohort study that used propensity score matching. After 3 and 6 months of therapy with Gla-300, there was significant reductions in mean $\mathrm{HbA}_{1 \mathrm{c}}$ by $17 \%$ and $28 \%$ respectively in male patients and by $18 \%$ and $29 \%$ respectively in male patients. 
Biochemical parameters (ACR, eGFR, FBS, PBS and $\mathrm{HbA}_{1 \mathrm{c}}$ ) from baseline to 3 months and 6 months post treatment were assessed using repeated measures of ANOVA and were found to be statistically significant. There was a reduction of $27 \%$ and $47 \%$ in albumin creatinine ratio from baseline to 3 months and 6 months respectively. Similarly the eGFR increased by $4 \%$ and $7 \%$ from baseline to 3 months and 6 months respectively. All glycemic control parameters decreased significantly with a $47 \%$ and $48 \%$ decline in FBS and PPBS from baseline to 6 months respectively. The $\mathrm{HbA}_{1 \mathrm{c}}$ decreased significantly from baseline to 3 months and 6 months post treatment by $17 \%$ and $29 \%$ respectively.

The majority of the patients i.e. 25 (50\%) achieved their individual $\mathrm{HbA}_{1 \mathrm{c}}$ target of $7 \%$ by switching their basal insulin to Gla-300, while $24(48 \%)$ achieved $\mathrm{HbA}_{1 \mathrm{c}}$ level of below $7.5 \%$.

Adequate dose titration is essential after the initiation of an insulin regimen after careful evaluation of the patient's glycemic control, but maybe delayed in clinical practice resulting in prolongation of the duration of diabetes and other comorbidities. Most important barrier for dose titration are concerns about hypoglycemia. First-generation basal insulin analogues such as Gla-100 were shown to provide significant advantages over NPH insulin regarding the risk for hypoglycemic events and administration, and recently approved second-generation basal insulins such as Gla-300 afford even greater improvements. This was illustrated in our study wherein a good number of patients were still maintained on the low dose of $0.2-0.3 \mathrm{IU} / \mathrm{kg}$ (89.5\%) and only 5 subjects required a dose titration to 0.4 units (10\%). The number of hypoglycaemia episodes were also not significant with only 5 subjects $(10.4 \%)$ reporting events none of which required hospitalization.

A major limitation of the current study was the small number of patients $(n=50)$ that were followed up until 6 months. The lack of a direct comparator is another major limitation, as no information on the outcomes with alternative lines of treatment could be compared. However, the study demonstrated consistent and sustained glucose control with Gla-300 and a significantly lower risk of hypoglycemia.

\section{Conclusion}

In patients with uncontrolled type 2 diabetes, switching from either OHAs or insulin to Gla-300 improves glycemic control, with a low incidence of hypoglycemia. These results confirm the effectiveness and safety of Gla-300 in a real-world setting and show that Gla-300 is a suitable basal insulin therapy option for patients with type 2 diabetes mellitus.

\section{Acknowledgments}

We would like to thank Dr. Monjori Mitra, Research Director and Liton Sarkar, Data Manager Medclin Research, Kolkata for providing data management and statistical analysis for this study.

\section{REFERENCES}

1. International Diabetes Federation. Brussels, Belgium: IDF; 2017. IDF diabetes atlas - 8th edition. https://idf.org/e-library/welcome. html. Accessed 10 Jun 2018.

2. American Diabetes Association. 1. Improving Care and Promoting Health in Populations - 2018. Diabetes Care. 2018; 41(Suppl 1): S7-SS12, doi: 10.2337/dc18-S001, indexed in Pubmed: 29222372.

3. Heise T, Mathieu C. Impact of the mode of protraction of basal insulin therapies on their pharmacokinetic and pharmacodynamic properties and resulting clinical outcomes. Diabetes Obes Metab. 2017; 19(1): 3-12, doi: 10.1111/dom.12782, indexed in Pubmed: 27593206.

4. US Food and Drug Administration. Approvalpackage.https://www. accessdata.fda.gov/scripts/cder/daf/index.cfm?event=overview. process\&AppINo $=206538$.

5. Riddle MC, Bolli GB, Ziemen $M$, et al. EDITION 1 Study Investigators. New insulin glargine 300 units/mL versus glargine 100 units $/ \mathrm{mL}$ in people with type 2 diabetes using basal and mealtime insulin: glucose control and hypoglycemia in a 6-month randomized controlled trial (EDITION 1). Diabetes Care. 2014; 37(10): 2755-2762, doi: 10.2337/dc14-0991, indexed in Pubmed: 25078900.

6. Yki-Järvinen $H$, Bergenstal $R$, Ziemen $M$, et al. EDITION 2 Study Investigators. New insulin glargine 300 units $/ \mathrm{mL}$ versus glargine 100 units $/ \mathrm{mL}$ in people with type 2 diabetes using oral agents and basal insulin: glucose control and hypoglycemia in a 6-month randomized controlled trial (EDITION 2). Diabetes Care. 2014; 37(12): 3235-3243, doi: 10.2337/dc14-0990, indexed in Pubmed: 25193531.

7. K/DOQI clinical practice guidelines for chronic kidney disease: Evaluation, classification, and stratification. Am J Kidney Dis. 2002; 39: S1-S266. 